\title{
Antibody Activity Against Mycobacterium leprae Antigen 7 in Leprosy: \\ Studies on Variation in Antibody Content Throughout the Spectrum and on the Effect of DDS Treatment and Relapse in BT Leprosy
}

\author{
LEO YODER, BEN NAAFS, MORTEN HARBOE* \\ and GUNNAR BJUNE
}

\begin{abstract}
All-Africa Leprosy and Rehabilitation Training Centre (ALERT) and Armauer Hansen Research Institute (AHRI), Addis Ababa, Ethiopia and University of Oslo, Institute for Experimental Medical Research, Ullevaal Hospital, Oslo 1, Norway
\end{abstract}

\begin{abstract}
Antibodies against Mycobacterium leprae antigen 7 were determined by a specific radioimmunoassay. The median value of 4 groups of patients decreased gradually from the lepromatous to the tuberculoid end of the spectrum, but there was a striking variation between the antibody content of individual sera in each group. Prolonged DDS treatment led to only a moderate decline of this antibody activity in lepromatous leprosy. In borderline tuberculoid leprosy, DDS treatment led to a marked decrease in antibody activity and a relapse is associated with renewed synthesis and increased antibody content.
\end{abstract}

\section{Introduction}

Research into the cellular immunity in leprosy has had major emphasis during the past few years (Myrvang et al., 1973; Bjune et al., 1976). Humoral immunity has also been studied, but to a lesser extent. Until recently, sensitive methods have not been applied to evaluate changes in its parameters during long term studies in leprosy.

* Requests for reprints should be addressed to Professor M. Harboe, Institute for Experimental Medical Research, Ullevaal Hospital, Oslo 1, Norway.

Received for publication 8 February, 1979.

$0305-7518 / 79 / 020113+09 \$ 02.00 / 0$

(c) 1979 British Leprosy Relief Association 
Rees et al. (1965) found a gradual fall in the amount of precipitating antibodies in lepromatous patients during DDS treatment. Melsom et al. (1978) used a specific radioimmunoassay for demonstration and quantitation of antibodies against Mycobacterium leprae antigen 7 and demonstrated a slight decline in the antibody activity in sera from lepromatous patients during the first year of DDS treatment. They noted that the fall in the antibody activity was small and contributed this to the enormous amount of antigen present in these lepromatous patients.

This paper describes our studies on antibodies against $M$. leprae antigen 7 throughout the clinical spectrum of leprosy. The effect of prolonged treatment with DDS was investigated in groups of patients with borderline tuberculoid (BT) leprosy with the intention of exploring whether changes in antibody activity against $M$. leprae antigen 7 could be used as an indicator of the effect of treatment or of relapse in tuberculoid leprosy.

\section{Materials and Methods}

Sera were obtained from patients attending the Addis Ababa Leprosy Hospital. The patients were classified clinically and in most cases histologically according to the Ridley Jopling scale (1966). For studies of antibody activity throughout the spectrum, sera were obtained from patients who were untreated or had been treated with DDS for less than half a year. The patients were divided into 4 groups; the LL group included patients with polar and subpolar lepromatous leprosy, the BT group included patients with BT and BT/TT leprosy.

The patients who were under treatment received DDS in doses of 50-100 mg daily.

To study the effect of prolonged DDS treatment in lepromatous leprosy, sera were obtained from 16 patients who had been treated with DDS for at least 10 years and whose skin smears had been negative for at least 5 years. Sera from 17 untreated lepromatous patients were tested for comparison.

To study the effect of DDS treatment in BT leprosy, antibody activity was determined in sera from groups of individuals. The control group consisted of sera from 18 patients with newly diagnosed BT leprosy. Sera were also obtained from 13 BT patients treated for half a year, 17 treated for 1 year, 11 treated for 2 years, and from 27 patients treated for 3 years or more. All these patients received DDS at the time the sera were obtained.

Another group of 7 patients had been "released from control" (RFC) which means that their disease had been considered to be cured after DDS treatment for at least 5-6 years, and they subsequently had no DDS medication for at least 2 years. The last group of 14 sera was obtained from BT patients released from control, but with clinically suspected or histologically proven relapses.

The sera were stored at $-25^{\circ} \mathrm{C}$ in order to be tested as a group towards the same labelled $M$. leprae antigen 7 preparation. The preparation and labelling of $M$. leprae antigen 7 and the radioimmunoassay were performed as described previously by Melsom et al. (1978). All sera were tested in dilutions $10^{-3}$ and 
$10^{-4}$. Comparison between groups gave the same overall results when these two dilutions were used. In the BT group, many sera from untreated patients gave low values at dilution $10^{-4}$; this dilution was thus less suitable for demonstration of decrease in antibody activity in BT patients. In lepromatous leprosy, however, many values are very high at dilution $10^{-3}$. In these figures, the findings at dilution $10^{-4}$ are therefore recorded for characterization of antibody activity throughout the spectrum and in lepromatous leprosy, whereas $10^{-3}$ is used for illustrating the effect of DDS treatment in tuberculoid leprosy. All values are given as mean values of double tests. To permit comparison between tests on different days, an internal standard was used and the activity expressed as radioactivity bound to staphylococci in per cent of the maximum binding activity by a reference serum pool containing strong anti- $M$. leprae antigen 7 activity.

The Wilcoxon test for two samples was used for calculation of statistical significance of the differences between groups of sera.

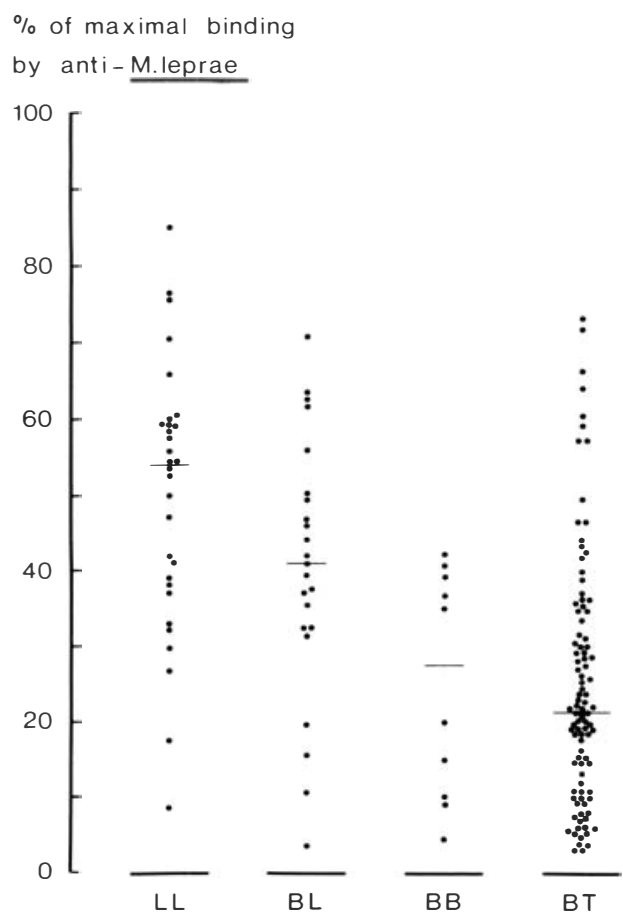

Fig. 1. Antibodies against $M$. leprae antigen 7 throughout the clinical spectrum of leprosy. Each point represents one patient. The activity is shown as binding of labelled antigen by serum diluted $10^{-4}$ and expressed as per cent of maximal binding by anti-M. leprae. The horizontal bars show median values in all figures. 


\section{Results}

Figure 1 shows anti- $M$. leprae antigen 7 activity in sera from patients throughout the clinical spectrum of leprosy. Two findings are apparent: the median value decreases gradually from the lepromatous to the tuberculoid end of the spectrum, and there is a striking variation between the antibody activity of individual sera within each of the four groups of patients. These findings confirm and extend our previous observations (Harboe et al., 1977; Melsom et al., 1978).

Analysis of the case records of the patients in the BT group showed that the antibody content was not significantly different in groups separated on the basis of sex, age and duration of disease. Neither did it appear to be correlated with the presence or absence of reaction, nor with the response to sonicated or whole $M$. leprae in the lymphocyte transformation test. Further data and analyses are needed to see whether there is a correlation with severity of the disease, e.g. as graded by a combined measure of bacillary content and extent of lesions.

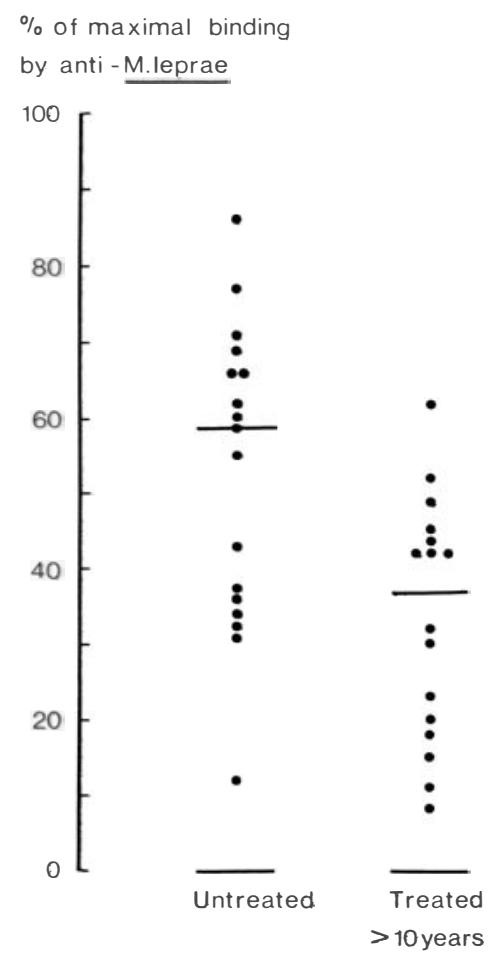

Fig. 2. Effect of prolonged DDS treatment on antibodies against $M$. leprae antigen 7 in lepromatous leprosy. Each point shows the activity in serum from one patient diluted $10^{-4}$. 
Figure 2 shows the findings in the two groups of patients with lepromatous leprosy. The median value at dilution $10^{-4}$ in the group of untreated patients is $59 \%$, with again a striking variation in the antibody content of the individual sera. In the group treated with DDS for over 10 years and with negative skin smears for at least 5 years, the antibody activity was lower, with a median value of $37 \%$. There was also a wide scatter between the individual sera in this group. The difference between the two groups is statistically significant $(P<0.005)$. At dilution $10^{-3}$, the median values were $87 \%$ and $75 \%$ respectively. The latter value is virtually the same as the median value of the group of untreated BT leprosy patients (74\%), shown in the extreme left column of Fig. 3.

Figure 3 shows the findings in the various groups of borderline tuberculoid (BT) leprosy, each point representing the activity in the serum from one patient diluted $10^{-3}$. The median value in the untreated group is $74 \%$ and there is a striking variation in antibody content in individual sera. The same variation is

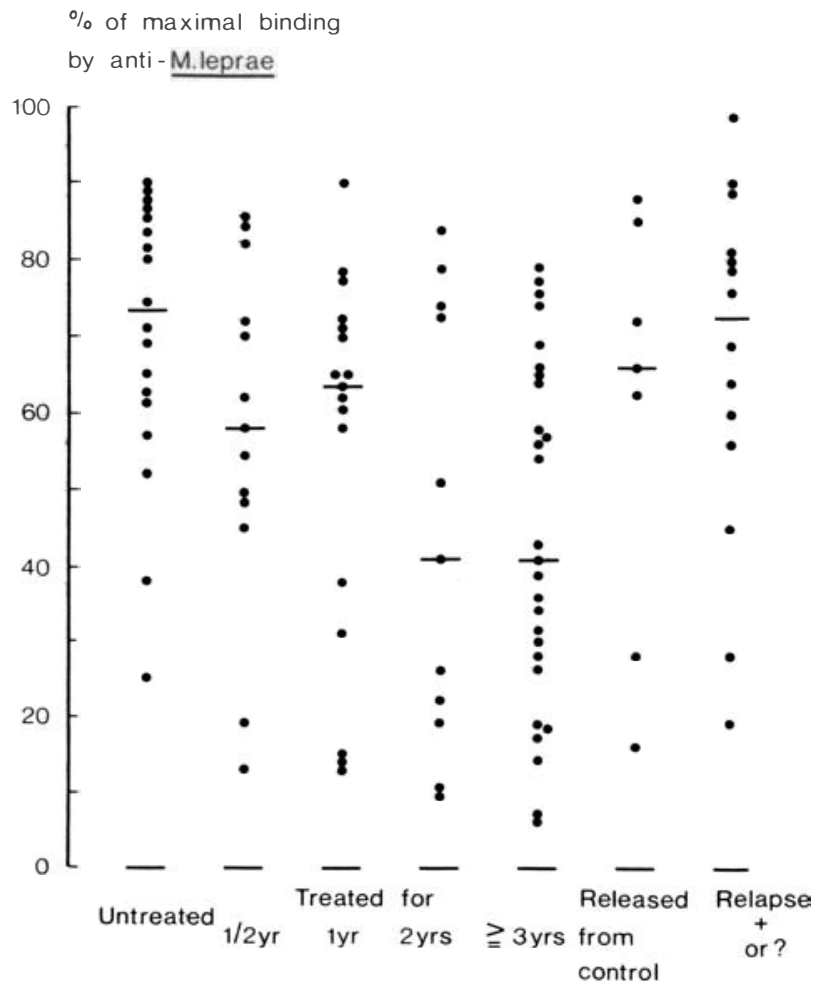

Fig. 3. Effect of DDS treatment and relapse on antibodies against $M$. leprae antigen 7 in borderline tuberculoid (BT) leprosy. Each point shows the activity in serum from one patient diluted $10^{-3}$. 
evident in the other groups, but the median value decreased markedly during DDS treatment to $41 \%$ in the groups who had been treated for 2 years and 3 years or more. The difference between the untreated group and the group treated for 3 years or more is statistically significant $(P<0.005)$. The median value of the group of RFC patients is higher, but the number of sera tested is small. The extreme right column shows the findings in sera from 14 patients released from control after prolonged DDS treatment, but who were seen at the hospital with a clinically suspected or histologically proven relapse. The antibody content varied markedly also within this group, but the median value was virtually identical to the untreated group. The difference between the group treated for 3 years or more and the group with relapse is statistically significant $(P<0.005)$.

\section{Discussion}

When the median values of distinct groups of patients are compared, there is a gradual decrease in antibody content against $M$. leprae antigen 7 from the lepromatous to the tuberculoid end of the leprosy spectrum (see Fig. 1). Within each group of patients, however, there was a striking variation in antibody content, both when distinct groups in the spectrum were tested and within groups of BT patients treated for different periods. These findings confirm and extend the previous findings of Melsom et al. (1978) and Harboe et al. (1977) who used a radioimmunoassay with labelled BCG antigen 60 which cross-reacts with $M$. leprae antigen 7 . The reason for this wide variation in antibody content within groups of patients with similar classifications based on clinical and histological criteria has not been established. It may be noted that studies of cellular immunity have provided similar results; the mean response in the lymphocyte transformation test, with $M$. leprae as the stimulating antigen, decreased continuously from the tuberculoid to the lepromatous end of the spectrum, but there was a wide variation within each of the groups close to the tuberculoid end (Myrvang et al., 1973).

A slight fall in antibody activity during DDS treatment of lepromatous leprosy was demonstrated by Rees et al. (1965) and Melsom et al. (1978). That the fall over a 10 year period or more is not more marked is indeed striking (see Fig. 1). In fact, we found that the median value of lepromatous leprosy patients treated for more than 10 years and with negative skin smears for more than 5 years was the same as for untreated BT patients. This can probably not be explained only on the basis of stimulation by cross-reacting antigens present in environmental mycobacteria. Since antibody production continues to be so high, a significant stimulus should be looked for and probably comes from persisting $M$. leprae in these lepromatous patients. Persisting, viable $M$. leprae have been demonstrated in such cases by Waters et al. (1974). Krieg and Meyers (1978) studied serial biopsy specimens from lepromatous leprosy patients during treatment. With effective chemotherapy, the bacilli became granular, and eventually lost their acid-fastness completely. When comparable sections, which contained no acid-fast bacilli, were stained 
by Gomori's methenamine silver technique, large numbers of bacilli were frequently detected. They concluded that these persisting carcases are probably a continuing source of $M$. leprae antigens long after the patients are considered bacteriologically negative by routine studies of skin smears. Another source of $M$. leprae antigen which cannot be ruled out is that a number of the lepromatous patients may harbour DDS-resistant bacilli. In Ethiopia, 3\% of these patients have developed DDS resistance each year during the last 4 years (Pearson et al., 1977).

In tuberculoid leprosy the bacillary load is much less, and it could be expected that effective DDS treatment together with the ability of the tuberculoid patient to dispose of the bacilli might lead to elimination of $M$. leprae antigen. This assumption seems to be confirmed by our results, showing a marked fall in anti- $M$. leprae antigen 7 activity during the first 3-4 years of DDS treatment. The possibility that DDS, as such, is responsible for the decrease in antibody content, must also be considered. However, we consider this explanation improbable. In DDS-treated lepromatous patients, the decrease occurs very slowly, and a high antibody content is seen in many patients even after more than 10 years of DDS treatment. In tuberculoid leprosy the variation between individual patients in the groups is as marked after 3 years of DDS treatment as in untreated patients, and this would not be expected if DDS had a marked suppressive effect on antibody production against $M$. leprae antigen 7 .

The demonstration of increased antibody content in the group of patients with suspected or proven relapse is important since it indicates that we may obtain a useful immunological indicator of relapse in tuberculoid leprosy. This is particularly valuable since criteria for effect of treatment and relapse are few in these patients with little or no detectable acid-fast bacilli in the skin lesions.

In relapse, with an increasing number of bacilli, increased antigenic content in the lesions is probably a very potent stimulus for antibody formation. These observations indicate that the occurrence of persisting $M$. leprae should not only be considered in lepromatous leprosy, but also in tuberculoid patients. Their number may be low, but they must be present as shown by the high relapse rate found by Touw-Langendijk and Naafs (1979) among borderline tuberculoid leprosy patients in Ethiopia, especially in the first 2 years after "release from control". It may be assumed that many BT patients who are considered to be clinically cured after DDS treatment and are released from control may harbour viable $M$. leprae which in most instances are adequately controlled by the host's immune system. If this fails, the infection will be reactivated. Duncan (1977) found an increased relapse rate after delivery in BT female patients who were released from control before pregnancy. This indicates that the persisting, previously dormant, bacilli start to multiply during pregnancy due to insufficient control by the immune system. This is analogous with endogeneous reactivation of tuberculosis (Ustvedt, 1947; Stead, 1965). A few of our patients may be DDS resistant since some of them who were released from control and then restarted on DDS treatment did not respond adequately, i.e. the disease was not arrested within a year ( $\mathrm{Naafs}$, unpublished observations). 
In this study, groups of patients, treated for different periods, were compared. Additional studies are now being carried out, in which blood samples are obtained from individual BT patients at regular intervals during DDS treatment to see if there is a gradual and regular fall in antibody content in patients who start with a high antibody concentration and respond clinically to treatment with DDS. In addition, serum is taken from BT patients when DDS treatment is terminated and stored at $-25^{\circ} \mathrm{C}$. New samples will be taken at a later date to permit simultaneous testing of the serum obtained at termination of treatment and later samples, to see whether an increased antibody content in individual patients is correlated with other criteria for relapse.

\section{Acknowledgements}

We would like to acknowledge the help and support of the staff of ALERT and AHRI during the study. We thank Ato Tadelle Gersion and Brit Sundsten for their excellent technical assistance.

Dr Yoder was supported by the American Leprosy Mission, The Leprosy Mission, and the Ethiopian Ministry of Health, Dr Naafs by the Netherlands Leprosy Relief Association and the Netherlands Government.

The work was supported by grants from Anders Jahre's Fund for the Promotion of Science, the Norwegian Council for Science and the Humanities, and by the Immunology of Leprosy (IMMLEP) component of the UNDP/World Bank/WHO Special Programme for Research and Training in Tropical Diseases.

\section{References}

Bjune, G., Barnetson, R. St C., Ridley, D. S. and Kronvall, G. (1976). Lymphocyte transformation test in leprosy; correlation of the response with inflammation of lesions. Clin. exp. Immun. 25, 85 .

Duncan, M. E. (1977). Effect of pregnancy on tuberculosis and leprosy. Paper presented at the 13th Annual Conference of the Ethiopian Medical Association.

Harboe, M., Closs, O., Bjorvatn, B. and Bjune, G. (1977). Antibodies against BCG-antigen 60 in mycobacterial inf ection. Brit. med.J. 2, 430.

Krieg, R. E. and Meyers, W. M. (1978). Demonstration of Mycobacterium leprae in tissues from bacteriologically negative treated lepromatous leprosy patients. Proc. XIth International Leprosy Congress, Mexico, D. F., 1978.

Melsom, R., Naafs, B., Harboe, M. and Closs, O. (1978). Antibody activity against Mycobacterium leprae antigen-7 during the first year of DDS treatment in lepromatous (BL-LL) leprosy. Lepr. Rev. 49, 17.

Myrvang, B., Godal, T., Ridley, D. S., Frøland, S. S. and Song, Y. K. (1973). Immune responsiveness to Mycobacterium leprae and other mycobacterial antigens throughout the clinical and histopathological spectrum of leprosy. Clin exp. Immun. 14, 541.

Pearson, J. M. H., Cap, J. A., Haile, G. S. and Rees, R. J. W. (1977). Dapsone-resistant leprosy and its implications for leprosy control programmes. Lepr. Rev. 48, 83.

Rees, R. J. W., Chatterjee, K. R., Pepys, J. and Tee, R. D. (1965). Some immunologic aspects of leprosy. Amer. Rev. resp. Dis. 92 (Suppl), 139.

Ridley, D. S. and Jopling, W. H. (1966). Classification of leprosy according to immunity. A five group system. Int. J. Lepr. 34, 255.

Stead, W. W. (1965). The pathogenesis of pulmonary tuberculosis among older persons. Amer. Rev. resp. Dis. $91,811$.

Touw-Langendijk, E. M. J. and Naafs, B. (1979). Relapse in leprosy after release from control. Lepr. Rev. 50, 123-127. 
Ustvedt, H. J. (1947). Pulmonary Tuberculosis and its Treatment, p. 47. Statles Press Ltd, London.

Waters, M. F. R., Rees, R. J. W., McDougall, A. C. and Weddell, A. G. M. (1974). Ten years of dapsone in lepromatous leprosy: clinical, bacteriological and histological assessment and the finding of viable leprosy bacilli. Lepr. Rev. 45, 288. 